Georgian Mathematical Journal

Volume 13 (2006), Number 4, 675-686

\title{
SUMS OF SQUARES AND SUMS OF TRIANGULAR NUMBERS
}

\author{
SHAUN COOPER AND MICHAEL HIRSCHHORN
}

Dedicated to the memory of G. Lomadze

\begin{abstract}
Motivated by two results of Ramanujan, we give a family of 15 results and 4 related ones. Several have interesting interpretations in terms of the number of representations of an integer by a quadratic form $\lambda_{1} x_{1}^{2}+$ $\cdots+\lambda_{m} x_{m}^{2}$, where $\lambda_{1}+\cdots+\lambda_{m}=2,4$ or 8 . We also give a new and simple combinatorial proof of the modular equation of order seven.
\end{abstract}

2000 Mathematics Subject Classification: Primary 11E25; Secondary 05A19, 11D85, 33D15.

Key words and phrases: Sum of squares, sum of triangular numbers, theta function, modular equation.

\section{INTRODUCTION}

Let $\psi(q)=\sum_{n=0}^{\infty} q^{n(n+1) / 2}$, where it is assumed that $|q|<1$. In Chapter 20 of his Second Notebook [8], S. Ramanujan gave the results

$$
\begin{aligned}
& \psi\left(q^{3}\right) \psi\left(q^{5}\right)-\psi\left(-q^{3}\right) \psi\left(-q^{5}\right)=2 q^{3} \psi\left(q^{2}\right) \psi\left(q^{30}\right), \\
& \psi(q) \psi\left(q^{15}\right)+\psi(-q) \psi\left(-q^{15}\right)=2 \psi\left(q^{6}\right) \psi\left(q^{10}\right) .
\end{aligned}
$$

The goal of this paper is to present 13 similar and 4 related formulas. Some of the formulas turn out to be modular equations of orders three and seven, and some have interesting combinatorial interpretations.

This work is organized as follows. The results are stated in Section 2. Section 3 is a discussion, and we give interpretations of some of the results in terms of the number of representations of an integer by a quadratic form. Proofs of the results are given in Section 4.

\section{Statement of Results}

Let $\tau$ be a complex number with positive imaginary part and define $q=$ $\exp (2 \pi i \tau)$. Let

$$
\eta(\tau)=q^{1 / 24} \prod_{n=1}^{\infty}\left(1-q^{n}\right), \quad \varphi(q)=\sum_{n=-\infty}^{\infty} q^{n^{2}}, \text { and } \psi(q)=\sum_{n=0}^{\infty} q^{n(n+1) / 2} .
$$

Let $\lambda=\left(\lambda_{1}, \ldots, \lambda_{m}\right)$ be a partition of a positive integer $k$. That is, $\lambda_{1}, \ldots, \lambda_{m}$ are integers which satisfy $\lambda_{1} \geq \cdots \geq \lambda_{m} \geq 1$ and $\lambda_{1}+\cdots+\lambda_{m}=k$. Let us 
define

$$
\begin{aligned}
& \varphi_{\lambda}(q)=\varphi\left(q^{\lambda_{1}}\right) \cdots \varphi\left(q^{\lambda_{m}}\right), \\
& \psi_{\lambda}(q)=q^{k / 8} \psi\left(q^{\lambda_{1}}\right) \cdots \psi\left(q^{\lambda_{m}}\right) .
\end{aligned}
$$

The following results were obtained by investigating the function $\psi_{\lambda}(q)+$ $\psi_{\lambda}(-q)$ for each partition of 8 . Not every partition of 8 yields a result. Theorem (2.4) is a restatement of Ramanujan's results given in the introduction.

\section{Theorem 2.1.}

$$
\begin{aligned}
\psi_{(1,1,1,1,1,1,1,1)}(q)+\psi_{(1,1,1,1,1,1,1,1)}(-q) & =16 \psi_{(1,1,1,1,1,1,1,1)}\left(q^{2}\right) \\
\psi_{(2,2,1,1,1,1)}(q)+\psi_{(2,2,1,1,1,1)}(-q) & =8 \psi_{(2,2,1,1,1,1)}\left(q^{2}\right) \\
\psi_{(3,3,1,1)}(q)+\psi_{(3,3,1,1)}(-q) & =4 \psi_{(3,3,1,1)}\left(q^{2}\right) \\
\psi_{(4,2,1,1)}(q)+\psi_{(4,2,1,1)}(-q) & =4 \psi_{(4,2,1,1)}\left(q^{2}\right) \\
\psi_{(7,1)}(q)+\psi_{(7,1)}(-q) & =2 \psi_{(7,1)}\left(q^{2}\right)
\end{aligned}
$$

Theorem 2.2.

$$
\begin{aligned}
& \psi_{(2,2,2,1,1)}(q)+\psi_{(2,2,2,1,1)}(-q)=4 \psi_{(4,1,1,1,1)}\left(q^{2}\right), \\
& \psi_{(4,1,1,1,1)}(q)+\psi_{(4,1,1,1,1)}(-q)=8 \psi_{(2,2,2,1,1)}\left(q^{2}\right) .
\end{aligned}
$$

\section{Theorem 2.3.}

$$
\begin{aligned}
& \psi_{(4,3,1)}(q)+\psi_{(4,3,1)}(-q)=2 \psi_{(6,1,1)}\left(q^{2}\right), \\
& \psi_{(6,1,1)}(q)+\psi_{(6,1,1)}(-q)=4 \psi_{(4,3,1)}\left(q^{2}\right) .
\end{aligned}
$$

Theorem 2.4.

$$
\begin{aligned}
\psi_{(5,3)}(q)+\psi_{(5,3)}(-q) & =2 \psi_{(15,1)}\left(q^{2}\right), \\
\psi_{(15,1)}(q)+\psi_{(15,1)}(-q) & =2 \psi_{(5,3)}\left(q^{2}\right) .
\end{aligned}
$$

Theorem 2.5.

$$
\begin{aligned}
\psi_{(3,3,2)}(q)+\psi_{(3,3,2)}(-q) & =4 \psi_{(12,3,1)}\left(q^{2}\right), \\
\psi_{(12,3,1)}(q)+\psi_{(12,3,1)}(-q) & =2 \psi_{(3,3,2)}\left(q^{2}\right) .
\end{aligned}
$$

In order to state the next theorem, it is convenient to make another definition. Let $\lambda=\left(\lambda_{1}, \ldots, \lambda_{m}\right)$ and $\mu=\left(\mu_{1}, \ldots, \mu_{\ell}\right)$ be partitions of any two positive integers. Let

$$
\psi_{\lambda \mid \mu}(q)=\frac{\psi_{\lambda}(q)}{\psi_{\mu}(q)}
$$

Theorem 2.6.

$$
\begin{aligned}
\psi_{(3,2,2,1)}(q)+\psi_{(3,2,2,1)}(-q) & =2 \psi_{(6,1,1,1,1) \mid(2)}\left(q^{2}\right), \\
\psi_{(6,1,1,1,1) \mid(2)}(q)+\psi_{(6,1,1,1,1) \mid(2)}(-q) & =8 \psi_{(3,2,2,1)}\left(q^{2}\right) .
\end{aligned}
$$

The last theorem involves the function $\varphi_{\lambda}(q)$. It is included because it leads to combinatorial properties which are similar to the ones that can be obtained from Theorem 2.1. 


\section{Theorem 2.7.}

$$
\begin{aligned}
\varphi_{(1,1,1,1)}(q)-\varphi_{(1,1,1,1)}\left(q^{2}\right) & =8 \psi_{(1,1,1,1)}\left(q^{2}\right)+16 \psi_{(1,1,1,1)}\left(q^{4}\right), \\
\varphi_{(3,1)}(q)-\varphi_{(3,1)}\left(q^{4}\right) & =2 \psi_{(3,1)}\left(q^{2}\right)+4 \psi_{(3,1)}\left(q^{8}\right), \\
\varphi_{(1,1)}(q)-\varphi_{(1,1)}\left(q^{2}\right) & =4 \psi_{(1,1)}\left(q^{4}\right), \\
\varphi_{(1)}(q)-\varphi_{(1)}\left(q^{4}\right) & =2 \psi_{(1)}\left(q^{8}\right) .
\end{aligned}
$$

\section{Discussion}

Many of the results in Theorems 2.1-2.7 have special significance. Some are equivalent to modular equations, and others have interesting combinatorial consequences. These are discussed in this section.

Let $\lambda=\left(\lambda_{1}, \ldots, \lambda_{m}\right)$ be a partition of $k$. Let us define coefficients $r_{\lambda}(n)$ and $t_{\lambda}(n)$ by

$$
\varphi_{\lambda}(q)=\sum_{n=0}^{\infty} r_{\lambda}(n) q^{n}, \quad q^{-k / 8} \psi_{\lambda}(q)=\sum_{n=0}^{\infty} t_{\lambda}(n) q^{n} .
$$

When $\lambda=(1, \ldots, 1)$ is the partition consisting of $k$ ones, we will write $r_{k}(n)$ and $t_{k}(n)$ for $r_{\lambda}(n)$ and $t_{\lambda}(n)$, respectively.

If $k$ is a multiple of 8 , let us define coefficients $s_{\lambda}(n)$ by

$$
\psi_{\lambda}(q)=\sum_{n=0}^{\infty} s_{\lambda}(n) q^{n} .
$$

We will use the convention that $r_{\lambda}(x), s_{\lambda}(x), t_{\lambda}(x), r_{k}(x)$ and $t_{k}(x)$ are all defined to be zero if $x$ is not a non-negative integer.

Observe that $r_{\lambda}(n)$ is the number of solutions of the equation

$$
\lambda_{1} x_{1}^{2}+\cdots+\lambda_{m} x_{m}^{2}=n
$$

in integers $x_{1}, \ldots, x_{m}$. Similarly, $t_{\lambda}(n)$ is the number of solutions of the equation

$$
\lambda_{1} \frac{x_{1}\left(x_{1}+1\right)}{2}+\cdots+\lambda_{m} \frac{x_{m}\left(x_{m}+1\right)}{2}=n
$$

in non-negative integers $x_{1}, \ldots, x_{m}$. Finally, in the case that $k$ is a multiple of $8, s_{\lambda}(n)$ is the number of solutions of the equation

$$
\lambda_{1} x_{1}^{2}+\cdots+\lambda_{m} x_{m}^{2}=8 n
$$

in positive odd integers $x_{1}, \ldots, x_{m}$, and

$$
s_{\lambda}\left(n+\frac{k}{8}\right)=t_{\lambda}(n) .
$$

We will also need the following facts, which are consequences of the Jacobi triple product identity.

$$
\begin{aligned}
\varphi(q) & =\frac{\eta(2 \tau)^{5}}{\eta(\tau)^{2} \eta(4 \tau)^{2}}, & \varphi(-q) & =\frac{\eta(\tau)^{2}}{\eta(2 \tau)}, \\
q^{1 / 8} \psi(q) & =\frac{\eta(2 \tau)^{2}}{\eta(\tau)}, & q^{1 / 8} \psi(-q) & =\frac{\eta(\tau) \eta(4 \tau)}{\eta(2 \tau)} .
\end{aligned}
$$


3.1. Discussion of Theorem 2.1 for $\lambda=(1,1,1,1,1,1,1,1)$. If we write each function in terms of $\eta(\tau), \eta(2 \tau)$ and $\eta(4 \tau)$, we see that Theorem 1 for the partition $\lambda=(1,1,1,1,1,1,1,1)$ is equivalent to each of the following four forms:

$$
\begin{aligned}
q \psi(q)^{8}-q \psi(-q)^{8} & =16 q^{2} \psi\left(q^{2}\right)^{8}, \\
\varphi\left(-q^{2}\right)^{8}-\varphi(-q)^{8} & =16 q \psi(-q)^{8}, \\
\varphi(q)^{4}-\varphi(-q)^{4} & =16 q \psi\left(q^{2}\right)^{4}, \\
\prod_{n=1}^{\infty}\left(1+q^{2 n-1}\right)^{8}-\prod_{n=1}^{\infty}\left(1-q^{2 n-1}\right)^{8} & =16 q \prod_{n=1}^{\infty}\left(1+q^{2 n}\right)^{8} .
\end{aligned}
$$

Equations (3.4) and (3.5) are classical, and were given in these forms by Ramanujan [1, p. 40] and Jacobi [6, p. 147], respectively. A simple proof of (3.4) using series manipulations was given in [7, p. 128].

Equation (3.3) implies that

$$
s_{\lambda}(n)=\frac{1}{16}\left(r_{\lambda}(n)-(-1)^{n / 2} r_{\lambda}(n / 2)\right) .
$$

Thus the study of the sequence $s_{\lambda}(n)$ is reduced to the study of the sequence $r_{\lambda}(n)$. From (3.3) we can also deduce

$$
\varphi(-q)^{8}=16 \sum_{j=0}^{\infty}-q^{\left(2^{j}\right)} \psi\left(-q^{\left(2^{j}\right)}\right)^{8} .
$$

Therefore

$$
r_{\lambda}(n)=16\left(s_{\lambda}(n)+(-1)^{n / 2} s_{\lambda}(n / 2)+(-1)^{n / 4} s_{\lambda}(n / 4)+\cdots\right) .
$$

Conversely, $r_{\lambda}(n)$ has been expressed as a finitely many terms of the form $s_{\lambda}\left(n / 2^{k}\right), k \geq 0$, and so the study of the sequence $r_{\lambda}(n)$ has been reduced to the study of the sequence $s_{\lambda}(n)$.

Equations (3.6) and (3.7) may be recast in more familiar terms as follows.

Proposition 3.1. Let $n$ be a positive integer, and write $n=2^{\alpha} k$, where $k$ is odd and $\alpha$ is a non-negative integer. Then

$$
\begin{aligned}
t_{8}(n-1) & =\frac{1}{16}\left(r_{8}(n)-(-1)^{n / 2} r_{8}(n / 2)\right), \\
r_{8}(n) & = \begin{cases}16 t_{8}(n-1), & \text { if } \alpha=0, \\
16 \sum_{j=1}^{\alpha} t_{8}\left(2^{j} k-1\right)-16 t_{8}(k-1), & \text { if } \alpha \geq 1 .\end{cases}
\end{aligned}
$$

Example 3.2. We have

$$
\begin{aligned}
\varphi(q)^{8}= & 1+16 q+112 q^{2}+448 q^{3}+1136 q^{4}+2016 q^{5}+3136 q^{6}+5504 q^{7} \\
& +9328 q^{8}+12112 q^{9}+14112 q^{10}+21312 q^{11}+31808 q^{12}+\cdots, \\
\psi(q)^{8}= & 1+8 q+28 q^{2}+64 q^{3}+126 q^{4}+224 q^{5}+344 q^{6}+512 q^{7}
\end{aligned}
$$




$$
+757 q^{8}+1008 q^{9}+1332 q^{10}+1792 q^{11}+2198 q^{12}+\cdots .
$$

Taking $n=12$ in Proposition 3.1, we obtain

$$
\begin{aligned}
& t_{8}(11)=\frac{1}{16}\left(r_{8}(12)-r_{8}(6)\right)=\frac{1}{16}(31808-3136)=1792, \\
& r_{8}(12)=16\left(t_{8}(11)+t_{8}(5)-t_{8}(2)\right)=16(1792+224-28)=31808 .
\end{aligned}
$$

If $n$ is odd, then Proposition 3.1 implies

$$
r_{8}(n)=16 t_{8}(n-1) .
$$

It would be interesting to have a direct combinatorial proof of this result.

3.2. Discussion of Theorem 2.1 for $\lambda=(3,3,1,1)$. If we follow the procedure in the previous section, we see that Theorem 1 for the partition $\lambda=$ $(3,3,1,1)$ is equivalent to each of the following four forms:

$$
\begin{aligned}
q \psi(q)^{2} \psi\left(q^{3}\right)^{2}-q \psi(-q)^{2} \psi\left(-q^{3}\right)^{2} & =4 q^{2} \psi\left(q^{2}\right)^{2} \psi\left(q^{6}\right)^{2}, \\
\varphi\left(-q^{2}\right)^{2} \varphi\left(-q^{6}\right)^{2}-\varphi(-q)^{2} \varphi\left(-q^{3}\right)^{2} & =4 q \psi(-q)^{2} \psi\left(-q^{3}\right)^{2}, \\
\varphi(q) \varphi\left(q^{3}\right)-\varphi(-q) \varphi\left(-q^{3}\right) & =4 q \psi\left(q^{2}\right) \psi\left(q^{6}\right), \\
\prod_{n=1}^{\infty}\left(1+q^{2 n-1}\right)^{2}\left(1+q^{6 n-3}\right)^{2} & \\
-\prod_{n=1}^{\infty}\left(1-q^{2 n-1}\right)^{2}\left(1-q^{6 n-3}\right)^{2} & =4 q \prod_{n=1}^{\infty}\left(1+q^{2 n}\right)^{2}\left(1+q^{6 n}\right)^{2} .
\end{aligned}
$$

These are all equivalent to the modular equation of order three:

$$
(\alpha \beta)^{1 / 4}+\{(1-\alpha)(1-\beta)\}^{1 / 4}=1,
$$

where $\beta$ has degree three over $\alpha$. See [1, p. 230-232], where both the modular equation and (3.10) are given. A simple proof of (3.10) which uses series manipulations was given in [2, p. 110]. Equation (3.11) was given in [4, p. 299].

Equation (3.9) implies that

$$
s_{\lambda}(n)=\frac{1}{4}\left(r_{\lambda}(n)-(-1)^{n / 2} r_{\lambda}(n / 2)\right) .
$$

Also from (3.9), we can deduce

$$
\varphi(-q)^{2} \varphi\left(-q^{3}\right)^{2}=4 \sum_{j=0}^{\infty}-q^{\left(2^{j}\right)} \psi\left(-q^{\left(2^{j}\right)}\right)^{2} \psi\left(-q^{3\left(2^{j}\right)}\right)^{2} .
$$

Therefore

$$
r_{\lambda}(n)=4\left(s_{\lambda}(n)+(-1)^{n / 2} s_{\lambda}(n / 2)+(-1)^{n / 4} s_{\lambda}(n / 4)+\cdots\right) .
$$

Thus, equation (3.12) reduces the study of $s_{\lambda}(n)$ to the study of $r_{\lambda}(n)$, and equation (3.13) shows the opposite is also true, namely, that the study of $r_{\lambda}(n)$ can be reduced to the study of $s_{\lambda}(n)$.

Equations (3.12) and (3.13) may be re-expressed as follows. 
Proposition 3.3. Let $n$ be a positive integer, and write $n=2^{\alpha} k$, where $k$ is odd and $\alpha$ is a non-negative integer. Then

$$
\begin{aligned}
& t_{\lambda}(n-1)= \frac{1}{4}\left(r_{\lambda}(n)-(-1)^{n / 2} r_{\lambda}(n / 2)\right), \\
& r_{\lambda}(n)= \begin{cases}4 t_{\lambda}(n-1), & \text { if } \alpha=0, \\
4 \sum_{j=1}^{\alpha} t_{\lambda}\left(2^{j} k-1\right)-4 t_{\lambda}(k-1), & \text { if } \alpha \geq 1 .\end{cases}
\end{aligned}
$$

If $n$ is odd, then Proposition 3.3 implies

$$
r_{\lambda}(n)=4 t_{\lambda}(n-1) .
$$

Once again, it would be interesting to have a direct combinatorial proof of this result.

3.3. Discussion of Theorem 2.1 for $\lambda=(7,1)$. If we follow the procedure in the previous sections, we see that Theorem 1 for the partition $\lambda=(7,1)$ is equivalent to each of the following four forms:

$$
\begin{aligned}
q \psi(q) \psi\left(q^{7}\right)-q \psi(-q) \psi\left(-q^{7}\right) & =2 q^{2} \psi\left(q^{2}\right) \psi\left(q^{14}\right), \\
\varphi\left(-q^{2}\right) \varphi\left(-q^{14}\right)-\varphi(-q) \varphi\left(-q^{7}\right) & =2 q \psi(-q) \psi\left(-q^{7}\right), \\
\sqrt{\varphi(q) \varphi\left(q^{7}\right)}-\sqrt{\varphi(-q) \varphi\left(-q^{7}\right)} & =2 q \sqrt{\psi\left(q^{2}\right) \psi\left(q^{14}\right)}, \\
\prod_{n=1}^{\infty}\left(1+q^{2 n-1}\right)\left(1+q^{14 n-7}\right) & \\
-\prod_{n=1}^{\infty}\left(1-q^{2 n-1}\right)\left(1-q^{14 n-7}\right) & =2 q \prod_{n=1}^{\infty}\left(1+q^{2 n}\right)\left(1+q^{14 n}\right) .
\end{aligned}
$$

These are all equivalent to the modular equation of order seven:

$$
(\alpha \beta)^{1 / 8}+\{(1-\alpha)(1-\beta)\}^{1 / 8}=1
$$

where $\beta$ has degree seven over $\alpha$. See $[1$, pp. 304, 314] where (3.15) and the modular equation are given. Equation (3.17) and an interesting combinatorial interpretation were given in [4, pp. 299, 482]. A simple proof of (3.17) using series manipulations has been given in [5]. We will give a bijective proof of (3.14), and hence a new proof of the modular equation of order seven, in Proposition 3.5 below.

Equation (3.15) implies that

$$
s_{\lambda}(n)=\frac{1}{2}\left(r_{\lambda}(n)-(-1)^{n / 2} r_{\lambda}(n / 2)\right) .
$$

Also from (3.15) we deduce [1, p. 304]

$$
\varphi(-q) \varphi\left(-q^{7}\right)=2 \sum_{j=0}^{\infty}-q^{\left(2^{j}\right)} \psi\left(-q^{\left(2^{j}\right)}\right) \psi\left(-q^{7\left(2^{j}\right)}\right) .
$$


Therefore

$$
r_{\lambda}(n)=2\left(s_{\lambda}(n)+(-1)^{n / 2} s_{\lambda}(n / 2)+(-1)^{n / 4} s_{\lambda}(n / 4)+\cdots\right) .
$$

Equations (3.18) and (3.19) may be re-expressed as follows.

Proposition 3.4. Let $n$ be a positive integer, and write $n=2^{\alpha} k$, where $k$ is odd and $\alpha$ is a non-negative integer. Then

$$
\begin{aligned}
& t_{\lambda}(n-1)= \frac{1}{2}\left(r_{\lambda}(n)-(-1)^{n / 2} r_{\lambda}(n / 2)\right), \\
& r_{\lambda}(n)= \begin{cases}2 t_{\lambda}(n-1), & \text { if } \alpha=0, \\
2 \sum_{j=1}^{\alpha} t_{\lambda}\left(2^{j} k-1\right)-2 t_{\lambda}(k-1), & \text { if } \alpha \geq 1 .\end{cases}
\end{aligned}
$$

If $n$ is odd, then Proposition 3.4 implies

$$
r_{\lambda}(n)=2 t_{\lambda}(n-1) .
$$

We will next give a direct combinatorial proof of the following result, which we then show is equivalent to (3.14).

Proposition 3.5. Let $n$ be a positive integer, and let $f(n)$ denote the number of solutions of the equation $x^{2}+7 y^{2}=8 n$ in odd integers. Then $f(n)=f(2 n)$.

Proof. We will refer to the equations $x^{2}+7 y^{2}=8 n$ and $u^{2}+7 v^{2}=16 n$ as Eq. (1) and Eq. (2), respectively.

Define a function $g:(2 \mathbb{Z}+1)^{2} \rightarrow(2 \mathbb{Z}+1)^{2}$ by

$$
g(x, y)=\left\{\begin{array}{lll}
((x-7 y) / 2,(x+y) / 2) & \text { if } x-y \equiv 0 \quad(\bmod 4), \\
((x+7 y) / 2,(y-x) / 2) & \text { if } x-y \equiv 2 \quad(\bmod 4) .
\end{array}\right.
$$

Let $(x, y)$ be a solution of Eq. (1) in odd integers, and put $(u, v)=g(x, y)$. Then

$$
u^{2}+7 v^{2}=\left(\frac{x \mp 7 y}{2}\right)^{2}+7\left(\frac{y \pm x}{2}\right)^{2}=2\left(x^{2}+7 y^{2}\right)=16 n
$$

and so $(u, v)$ is a solution of Eq. (2) in odd integers. Furthermore, by checking the two cases separately, we find that $u-v \equiv x-y(\bmod 4)$.

We will show that every solution of Eq. 2 arises this way. The inverse function $g^{-1}:(2 \mathbb{Z}+1)^{2} \rightarrow \mathbb{Z}^{2}$ is given by

$$
g^{-1}(u, v)=\left\{\begin{array}{lll}
((u+7 v) / 4,(v-u) / 4) & \text { if } u-v \equiv 0 \quad(\bmod 4) \\
((u-7 v) / 4,(u+v) / 4) & \text { if } u-v \equiv 2 \quad(\bmod 4)
\end{array}\right.
$$

Let $(u, v)$ be a solution of Eq. (2) in odd integers, and let $(x, y)=g^{-1}(u, v)$. It is straightforward to check that that $(x, y)$ is a solution of Eq. (1) in integers. It remains to show that $x$ and $y$ are both odd. If we consider $u$ and $v$ modulo 8 and write

$$
u \equiv j \quad(\bmod 8), \quad v \equiv k \quad(\bmod 8), \quad \text { where } j, k \in\{1,3,5,7\},
$$


then the condition $u^{2}+7 v^{2} \equiv 0(\bmod 16)$ implies that

$$
(j, k) \in\{(1,3),(3,1),(1,5),(5,1),(3,7),(7,3),(5,7),(7,5)\} .
$$

It follows that for the case $u \equiv v(\bmod 4)$, we have $(j, k) \in\{(1,5),(5,1)$, $(3,7),(7,3)\}$, and therefore $x=(u+7 v) / 4 \equiv 1(\bmod 2)$ and $y=(v-$ $u) / 4 \equiv 1(\bmod 2)$. Similarly, for the case $u \equiv v+2(\bmod 4)$, we have $(j, k) \in$ $\{(1,3),(3,1),(5,7),(7,5)\}$, and therefore $x=(u-7 v) / 4 \equiv 1(\bmod 2)$ and $y=(u+v) / 4 \equiv 1(\bmod 2)$. Thus $(x, y)$ is a solution of Eq. (1) in odd integers, as claimed.

Proposition 3.5 may be shown to be equivalent to (3.14) as follows. First, we have

$$
\begin{aligned}
\sum_{n=1}^{\infty} f(n) q^{n} & =\sum_{n=1}^{\infty} \sum_{\substack{x, y \text { odd } \\
x^{2}+7 y^{2}=8 n}} q^{\left(x^{2}+7 y^{2}\right) / 8} \\
& =\sum_{j=-\infty}^{\infty} q^{(2 j+1)^{2} / 8} \sum_{k=-\infty}^{\infty} q^{7(2 k+1)^{2} / 8}=4 q \psi(q) \psi\left(q^{7}\right)
\end{aligned}
$$

This together with Proposition 3.5 implies

$$
\begin{aligned}
q \psi(q) \psi\left(q^{7}\right)-q \psi(-q) \psi\left(-q^{7}\right) & =\frac{1}{2} \sum_{n=1}^{\infty} f(2 n) q^{2 n}=\frac{1}{2} \sum_{n=1}^{\infty} f(n) q^{2 n} \\
& =2 q^{2} \psi\left(q^{2}\right) \psi\left(q^{14}\right) .
\end{aligned}
$$

Therefore we have obtained (3.14) from Proposition 3.5. The procedure can be reversed, to yield Proposition 3.5 from (3.14).

3.4. Discussion of Theorem 2.7 for $\lambda=(1,1,1,1)$. Theorem 2.7 in this instance is equivalent to

$$
\varphi(q)^{4}-\varphi\left(q^{2}\right)^{4}=8 q \psi\left(q^{2}\right)^{4}+16 q^{2} \psi\left(q^{4}\right)^{4} .
$$

If we compare the coefficients of $q^{2 n+1}$ on both sides, we obtain

$$
t_{4}(n)=\frac{1}{8} r_{4}(2 n+1)
$$

Next, if we replace $q$ by $q^{\left(2^{j}\right)}$ in (3.20) and sum over all non-negative integers $j$, we obtain

$$
\varphi(q)^{4}=1+8 q \psi\left(q^{2}\right)^{4}+24 \sum_{j=1}^{\infty} q^{\left(2^{j}\right)} \psi\left(q^{\left(2^{j+1}\right)}\right)^{4} .
$$

Compare the coefficients of $q^{n}$ to obtain

$$
r_{4}(n)=8 t_{4}\left(\frac{n-1}{2}\right)+24 \sum_{j=1}^{\infty} t_{4}\left(\frac{n-2^{j}}{2^{j+1}}\right) .
$$


Only one of the numbers $\left(n-2^{j}\right) / 2^{j+1}$, where $j \geq 0$, is an integer, hence we deduce, for any non-negative integers $\alpha$ and $k$, that

$$
r_{4}\left(2^{\alpha}(2 k+1)\right)= \begin{cases}8 t_{4}(k) & \text { if } \alpha=0 \\ 24 t_{4}(k) & \text { if } \alpha \geq 1\end{cases}
$$

Equation (3.21) shows that $t_{4}(n)$ can be obtained from a subsequence of $r_{4}(n)$, and (3.22) shows that conversely, $r_{4}(n)$ can be obtained from a subsequence of $t_{4}(n)$.

3.5. Discussion of Theorem 2.7 for $\lambda=(3,1)$. Theorem 2.7 in this instance is equivalent to

$$
\varphi(q) \varphi\left(q^{3}\right)-\varphi\left(q^{4}\right) \varphi\left(q^{12}\right)=2 q \psi\left(q^{2}\right) \psi\left(q^{6}\right)+4 q^{4} \psi\left(q^{8}\right) \psi\left(q^{24}\right) .
$$

If we compare the coefficients of $q^{2 n+1}$ on both sides, we obtain

$$
t_{\lambda}(n)=\frac{1}{2} r_{\lambda}(2 n+1) \text {. }
$$

Next, if we replace $q$ by $q^{\left(2^{2 j}\right)}$ in (3.23) and sum over all non-negative integers $j$, we obtain

$$
\varphi(q) \varphi\left(q^{3}\right)=1+2 q \psi\left(q^{2}\right) \psi\left(q^{6}\right)+6 \sum_{j=1}^{\infty} q^{\left(2^{2 j+1}\right)} \psi\left(q^{\left(2^{2 j+1}\right)}\right) \psi\left(q^{\left(3 \times 2^{2 j+1}\right)}\right) .
$$

Compare the coefficients of $q^{n}$ to obtain

$$
r_{\lambda}(n)=2 t_{\lambda}\left(\frac{n-1}{2}\right)+6 \sum_{j=1}^{\infty} t_{\lambda}\left(\frac{n-2^{2 j}}{2^{2 j+1}}\right) .
$$

At most one of the numbers $\left(n-2^{2 j}\right) / 2^{2 j+1}$, where $j \geq 0$, can be an integer, hence we deduce, for any non-negative integers $\alpha$ and $k$, that

$$
r_{\lambda}\left(2^{\alpha}(2 k+1)\right)= \begin{cases}2 t_{\lambda}(k) & \text { if } \alpha=0 \\ 6 t_{\lambda}(k) & \text { if } \alpha \neq 0 \text { is even } \\ 0 & \text { if } \alpha \text { is odd }\end{cases}
$$

Equation (3.24) shows that $t_{\lambda}(n)$ can be obtained from a subsequence of $r_{\lambda}(n)$, and (3.25) shows that conversely, $r_{\lambda}(n)$ can be obtained from a subsequence of $t_{\lambda}(n)$.

3.6. Discussion of Theorem 2.7 for $\lambda=(1,1)$. Theorem 2.7 in this instance is equivalent to

$$
\varphi(q)^{2}-\varphi\left(q^{2}\right)^{2}=4 q \psi\left(q^{4}\right)^{2} .
$$

If we compare the coefficients of $q^{4 n+1}$ on both sides, we obtain

$$
t_{2}(n)=\frac{1}{4} r_{2}(4 n+1) \text {. }
$$


Next, if we replace $q$ by $q^{\left(2^{j}\right)}$ in (3.23) and sum over all non-negative integers $j$, we obtain

$$
\varphi(q)^{2}=1+4 \sum_{j=0}^{\infty} q^{\left(2^{j}\right)} \psi\left(q^{\left(2^{j+2}\right)}\right)^{2} .
$$

Compare the coefficients of $q^{n}$ to obtain

$$
r_{2}(n)=4 \sum_{j=0}^{\infty} t_{\lambda}\left(\frac{n-2^{j}}{2^{j+2}}\right) .
$$

The number $\left(n-2^{j}\right) / 2^{j+2}$, where $j \geq 0$, will be an integer if and only if $n=2^{j}(4 k+1)$ for some non-negative integer $k$. Hence we deduce that

$$
r_{2}(n)= \begin{cases}4 t_{2}(k) & \text { if } n=2^{j}(4 k+1) \\ & \text { for some non-negative } \\ 0 & \text { integers } j \text { and } k,\end{cases}
$$

Equation (3.27) shows that $t_{2}(n)$ can be obtained from a subsequence of $r_{2}(n)$, and (3.28) shows that conversely, $r_{2}(n)$ can be obtained from a subsequence of $t_{2}(n)$.

3.7. Discussion of Theorem $\mathbf{2 . 7}$ for $\lambda=(1)$. The analysis of Theorem 2.7 in this instance is trivial. We obtain the results:

(1) The positive integer $n$ is a triangular number if $8 n+1$ is a square.

(2) The positive integer $n$ is a square if $n=2^{2 j}(8 k+1)$ for non-negative integers $j$ and $k$, and $k$ is a triangular number.

\section{Proofs}

In this section we will give the proofs of Theorems 2.1-2.7. We will require the following lemma.

Lemma 4.1.

$$
\begin{aligned}
\varphi(q) \psi\left(q^{2}\right) & =\psi(q)^{2}, \\
\psi(q)^{2}+\psi(-q)^{2} & =2 \varphi\left(q^{4}\right) \psi\left(q^{2}\right), \\
\psi(q)^{2}-\psi(-q)^{2} & =4 q \psi\left(q^{2}\right) \psi\left(q^{8}\right), \\
\psi(q)^{4}-\psi(-q)^{4} & =8 q \psi\left(q^{2}\right)^{2} \psi\left(q^{4}\right)^{2}, \\
\psi(q) \psi\left(q^{3}\right)-\psi(-q) \psi\left(-q^{3}\right) & =2 q \varphi\left(q^{2}\right) \psi\left(q^{12}\right), \\
\psi(q) \psi\left(q^{3}\right)+\psi(-q) \psi\left(-q^{3}\right) & =2 \varphi\left(q^{6}\right) \psi\left(q^{4}\right) .
\end{aligned}
$$

Proof. The first part follows from the eta-quotients in (3.1). Next, we have

$$
\psi(q)^{2}=\varphi(q) \psi\left(q^{2}\right)=\left(\varphi\left(q^{4}\right)+2 q \psi\left(q^{8}\right)\right) \psi\left(q^{2}\right) .
$$

The second and third parts follow from this by replacing $q$ by $-q$ and adding or subtracting. The fourth part follows by multiplying the second and third parts and simplifying the result using the first part. The last two parts follow from [3, (xxxiii)]. 
4.1. Proof of Theorem 2.1. References to proofs for $\lambda=(1,1,1,1,1,1,1,1)$, $(3,3,1,1)$, and $(7,1)$ were given in the previous section. We will give the proofs for the remaining two cases $\lambda=(2,2,1,1,1,1)$ and $(4,2,1,1)$.

For $\lambda=(2,2,1,1,1,1)$, we have, using the fourth part of Lemma 4.1,

$$
\begin{aligned}
& \psi_{\lambda}(q)+\psi_{\lambda}(-q) \\
& \quad=q \psi\left(q^{2}\right)^{2}\left(\psi(q)^{4}-\psi(-q)^{4}\right)=8 q^{2} \psi\left(q^{4}\right)^{2} \psi\left(q^{2}\right)^{4}=8 \psi_{\lambda}\left(q^{2}\right) .
\end{aligned}
$$

For $\lambda=(4,2,1,1)$, we have, using the third part of Lemma 4.1,

$$
\begin{aligned}
& \psi_{\lambda}(q)+\psi_{\lambda}(-q) \\
& \quad=q \psi\left(q^{4}\right) \psi\left(q^{2}\right)\left(\psi(q)^{2}-\psi(-q)^{2}\right)=4 q^{2} \psi\left(q^{8}\right) \psi\left(q^{4}\right) \psi\left(q^{2}\right)^{2}=4 \psi_{\lambda}\left(q^{2}\right) .
\end{aligned}
$$

4.2. Proof of Theorem 2.2 and 2.3. The proofs are similar to the proofs just given for the partitions $(2,2,1,1,1,1)$ and $(4,2,1,1)$. We omit the details.

4.3. Proof of Theorem 2.4. These results were stated by Ramanujan and proved in [1, pp. 377-378].

4.4. Proof of Theorem 2.5. By the third part of Lemma 4.1 with $q^{3}$ in place of $q$, we obtain

$$
\begin{aligned}
& \psi_{(3,3,2)}(q)+\psi_{(3,3,2)}(-q) \\
& \quad=q \psi\left(q^{2}\right)\left(\psi\left(q^{3}\right)^{2}-\psi\left(-q^{3}\right)^{2}\right)=4 q^{2} \psi\left(q^{2}\right) \psi\left(q^{6}\right) \psi\left(q^{24}\right)=4 \psi_{(12,3,1)}\left(q^{2}\right) .
\end{aligned}
$$

Next, using the first and last parts of Lemma 4.1, we find

$$
\begin{aligned}
\psi_{(12,3,1)}(q)+\psi_{(12,3,1)}(-q) & =q^{2} \psi\left(q^{12}\right)\left(\psi(q) \psi\left(q^{3}\right)+\psi(-q) \psi\left(-q^{3}\right)\right) \\
& =2 q^{2} \psi\left(q^{12}\right) \varphi\left(q^{6}\right) \psi\left(q^{4}\right) \\
& =2 q^{2} \psi\left(q^{6}\right)^{2} \psi\left(q^{4}\right) \\
& =2 \psi_{(3,3,2)}\left(q^{2}\right) .
\end{aligned}
$$

4.5. Proof of Theorem 2.6. The proof is similar to the proofs already given, so we omit the details.

4.6. Proof of Theorem 2.7. We will prove the results in the reverse order.

Proof for $\lambda=(1)$ : Add parts (i) and (ii) of Entry 25 in [1, p. 40].

Proof for $\lambda=(1,1)$ : Add parts (v) and (vi) of Entry 25 in [1, p. 40].

Proof for $\lambda=(3,1)$ : We have

$$
\begin{aligned}
& \varphi(q) \varphi\left(q^{3}\right) \\
& =\sum_{m, n=-\infty}^{\infty} q^{m^{2}+3 n^{2}} \\
& =\sum_{m, n=-\infty}^{\infty} q^{\left((m+3 n)^{2}+3(m-n)^{2}\right) / 4} \\
& =\sum_{u \equiv v} q^{\left(u^{2}+3 v^{2}\right) / 4}
\end{aligned}
$$




$$
\begin{aligned}
& =\sum_{k, l=-\infty}^{\infty} q^{4 k^{2}+12 l^{2}}+2 q \sum_{k, l=-\infty}^{\infty} q^{4 k^{2}+2 k+12 l^{2}+6 l}+q^{4} \sum_{k, l=-\infty}^{\infty} q^{4 k^{2}+4 k+12 l^{2}+12 l} \\
& =\varphi\left(q^{4}\right) \varphi\left(q^{12}\right)+2 q \psi\left(q^{2}\right) \psi\left(q^{6}\right)+4 q^{4} \psi\left(q^{8}\right) \psi\left(q^{24}\right) .
\end{aligned}
$$

Proof for $\lambda=(1,1,1,1)$ : From the result for $\lambda=(1,1)$ we have

$$
\varphi(q)^{4}=\left(\varphi\left(q^{2}\right)^{2}+4 q \psi\left(q^{4}\right)^{2}\right)^{2}=\varphi\left(q^{2}\right)^{4}+8 q \varphi\left(q^{2}\right)^{2} \psi\left(q^{4}\right)^{2}+16 q^{2} \psi\left(q^{4}\right)^{4} .
$$

Now use the first part of Lemma 4.1 on the middle term to complete the proof.

\section{REFERENCES}

1. B. C. Berndt, Ramanujan's notebooks. Part III. Springer-Verlag, New York, 1991.

2. J. M. Borwein and P. B. Borwein, Pi and the AGM. A study in analytic number theory and computational complexity. Canadian Mathematical Society Series of Monographs and Advanced Texts. A Wiley-Interscience Publication. John Wiley \& Sons, Inc., New York, 1987.

3. S. Cooper and M. D. Hirschhorn, Results of Hurwitz type for three squares. Discrete Math. 274(2004), No. 1-3, 9-24.

4. H. M. FARKAS and I. KRA, Theta constants, Riemann surfaces and the modular group. An introduction with applications to uniformization theorems, partition identities and combinatorial number theory. Graduate Studies in Mathematics, 37. American Mathematical Society, Providence, RI, 2001.

5. M. D. HirschHorn, The case of the mysterious sevens. Int. J. Number Theory 2(2006), No. 2, 213-216.

6. C. G. J. JACOBI, Gesammelte Werke. I. Herausgegeben auf Veranlassung der Königlich Preussischen Akademie der Wissenschaften. Zweite Ausgabe Chelsea Publishing Co., New York, 1969.

7. H. MCKean and V. Moll, Elliptic curves. Function theory, geometry, arithmetic. Cambridge University Press, Cambridge, 1997.

8. S. Ramanujan, Notebooks. Vols. 1, 2. Tata Institute of Fundamental Research, Bombay, 1957.

(Received 20.10.2006)

Authors' addresses:

S. Cooper

Institute of Information and Mathematical Sciences

Massey University

Private Bag 102904, North Shore Mail Centre, Auckland

New Zealand

E-mail: s.cooper@massey.ac.nz

M. Hirschhorn

School of Mathematics and Statistics

University of New South Wales

Sydney 2052, Australia

E-mail: m.hirschhorn@unsw.edu.au 